\title{
Stellungnahmeverfahren zur MTR-Ausbildungs- und Prüfungsverordnung
}

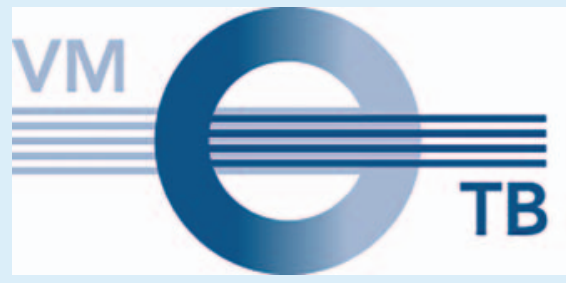

Nachdem Anfang dieses Jahres das neue MTA-Reformgesetz verabschiedet wurde, erarbeitet das Bundesgesundheitsministerium (BMG) hierfür gerade eine neue Ausbildungsund Prüfungsverordnung für Medizinische Technologen/Technologinnen (APrV). Diese soll noch in dieser Wahlperiode beschlossen werden. Damit wird die bisherige APrV nach vielen Jahren endlich novelliert. Dies haben wir gemeinsam mit der DRG und dem DVTA bereits seit Jahren gefordert. Die neue APrV soll 2023 in Kraft treten.

Die neue APrV gibt den Schulen viel eigenen Handlungsspielraum. Während in der bisher gültigen Fassung von 1994 noch detaillierte Vorgaben zu Inhalten gemacht wurden, entfällt dies in dieser Form. In der vorliegenden Entwurfsfassung sollen in erster Linie Kompetenzen im Bereich der Radiologie, Strahlentherapie und Nuklearmedizin, aber auch im Bereich der interdisziplinären Arbeit im Team und im Umgang mit Patienten/Patientinnen vermittelt werden. Wie dies von den Schulen im Einzelnen umgesetzt wird, bleibt abzuwarten. In jedem Fall ermöglicht der Fokus auf Kompe- tenzen eine schnellere Anpassung an neue Entwicklungen in der Medizin und im klinischen Alltag als bisher.

Die VMTB hat gemeinsam mit DRG und GPR eine Stellungnahme an das BMG übermittelt und sich hierfür auch mit weiteren Fachverbänden abgestimmt.

Die gemeinsame Stellungnahme der VMTB, DRG und GPR und die Entwurfsfassung des BMG können Sie einsehen unter: www.vmtb.de. 